\title{
Application of Computer 3D Digital Technology in Surgical Treatment of Pediatric Skull Deformity
}

\author{
Li Xiang ${ }^{1}$, Lu Zheng ${ }^{1}$, Li Zhicen ${ }^{1}$, Zhu Wanchun ${ }^{1}$, He Jintao ${ }^{1}$, Yu Yaxiong ${ }^{1}$, Gong Jian ${ }^{1}{ }^{*}$ \\ ${ }^{1}$ Beijing Tiantan Hospital Affiliated to Capital Medical University, Beijing 100070, China
}

\begin{abstract}
Pediatric skull deformity requires immediate surgery as indicated by increased cranial pressure, mental retardation, impaired or absent vision, cranial deformity, and mental and spiritual defects. This study explores the application value of computer aided simulation in treatment of pediatric skull deformity. The application of computer simulation surgery in the treatment of children with pediatric skull deformity allows surgeons to be familiar with the operation process in advance. The use of computer $3 \mathrm{D}$ digital technology for preoperative design planning and simulation can reduce surgical difficulty to a certain extent, improve surgical efficiency, significantly increase intraoperative accuracy, and also reduce the risk of intraoperative bleeding and postoperative complications.
\end{abstract}

\section{Main causes of pediatric skull deformity}

Pediatric skull deformity is a result of various factors, the most common of which is craniosynostosis. Craniosynostosis is skull deformity caused by premature closure of the cranial suture which results in skull development disorder. Cranial sutures at different locations have different probabilities of premature closure. Some studies have shown that the incidence from high to low is premature closure of sagittal suture (40\%-50\%), premature closure of coronary suture (20\%$25 \%)$, frontal premature closure $(5 \%-15 \%)$ and premature closure of lambdoidal suture (1\%-5\%) [1]. Premature closure at different positions usually leads to deformities in different shapes. For instance: sagittal suture can cause scaphoid cranial deformity; premature closure of coronal suture can cause brachycephaly, and there will be oxycephaly if it is combined with premature closure of other cranial suture; premature closure of coronal suture on one side and sphenofrontal suture generally results in frontal plagiocephaly; premature closure of lambdoidal suture on one side results in occipital plagiocephaly; premature closure of coronal suture, squamous suture, lambdoidal suture on side generally causes hemi-plagiocephaly; premature closure of lambdoidal suture will cause flattened posterior skull base and thickened occipital bone. According to studies by Virchow et al., after premature closure of the cranial suture, due to the limited growth of skulls in some part and too fast compensatory growth of other parts, there will be various skull deformities [2].

The growth and development of the skull and the closure process of the sutura are mainly controlled by brain development signals from the dura mater and ectoderm, while growth factors play a key role in the process of brain development signal transduction [3]. The release of various signaling factors is subject to joint regulation by the developing skull, cranial suture, dura mater and brain. Sutura mesenchyma is subject to combined action of various signaling factors, which results in consistent growth and development of the skull and the intracranial brain. Once genetic factors or the microenvironment of the cranial suture has changed, release and regulation of signal factors will be intervened, thereby leading to diseases like craniosynostosis or cranioschisis.

\section{Main treatment approaches for pediatric skull deformity}

In addition to cranial deformity, the clinical manifestations of craniosynostosis also include accompanying symptoms of increased intracranial pressure, hydrocephalus, visual impairment and proptosis, plus mental disorders. The clinical manifestations of craniosynostosis differ depending on premature closure of different cranial sutures, but the main treatment purpose is to expand the cranial cavity to increase the brain development space for better shape, etc. [4].

So far, the treatment of craniosynostosis has been limited to surgical treatment. However, regarding the choice of surgical timing, as well as the surgical methods and procedures, there has been no completely consistent view in academia and clinical practice. Some researchers believe that the best time for surgery is 6 months after 
birth [5]. Tessier and Marchac et al. proposed the need for cranium reconstruction during surgery, so that the cranial cavity can be constructed beforehand without reliance on brain development[6]. Cranioplasty reconstruction surgery usually has the following types: cranial suture reconstruction, total cranial suture reconstruction, metopoplasty of trigonocephalia, frontoorbital advancement, fronto-orbital advancement and orbital reconstruction, monobloc advancement, etc. In recent years, scholars at home and abroad have continuously proposed various new or improved surgical procedures [7 10].

\section{Computer-aided design for pediatric skull deformity surgery}

There are many surgical procedures in the treatment of craniosynostosis, but to do that procedures generally with great operation difficulty and high risk, and the postoperative effect is often not particularly satisfactory. With the rapid development of computer technology, especially computer graphics technology, computer 3D digital technology has also been applied to craniofacial surgery [11]. Computer 3D digital technology not only provides more accurate assessment method for the diagnosis of craniofacial deformities, but also provides a powerful tool for studying quantitative laws of craniofacial deformities. Through computer 3D digital technology, preoperative surgical simulation design can be performed to optimize the surgical plan, enhance doctor-patient communication, and thereby develop a personalized surgical plan to maximize the surgical efficiency and reduce surgical difficulty to a certain extent.

The author applied 3D assisted design software IVSP-Image developed by Beijing Ivsplan Technology Development Company in clinical practice to carry out surgical design and surgical path optimization for cranial deformity. This software features real-time 3D rendering and user-friendly operation mode, as well as abundant and easy-to-use cephalometric and 3D measurement tools.

The following are the main steps of surgical design using IVSP-Image software:

\section{Step 1. 3D reconstruction}

Import CT data on the skull into the IVSP-Image software, select $\mathrm{Hu}$ value of DICOM image to reconstruct the skull model. In the process of $3 \mathrm{D}$ reconstruction, one can also select points of interest. If points of interest are selected, the software will perform $3 \mathrm{D}$ reconstruction of the part connected with points of interest via connected region algorithm, and discard all the parts not connected to the point of interest. As IVSPImage adopts accelerated marching cube algorithm [1214], it can provide real-time 3D reconstruction function and real-time model editing and erasure function. Users can easily erase unnecessary parts on the 3D model, or erase unnecessary parts from the MPR surface of Dicom's original data. In addition, in the process of $3 \mathrm{D}$ reconstruction, morphological algorithm and 3D linear sampling algorithm are taken to preprocess the original
Dicom data, which allows smoother reconstructed model, as shown in Fig.1.

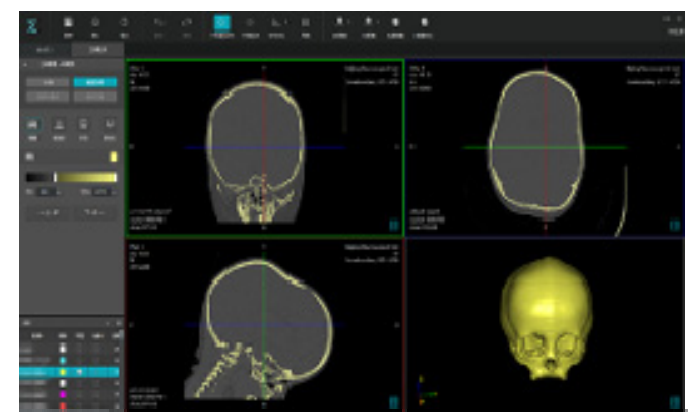

Figure 1 shows the 3D skull model reconstructed by the author in practice

\section{Step 2. Measurement}

After reconstruction of 3D skull model, it is possible to observe the model from all angles in the software via $\mathrm{zoom} / \mathrm{pan} /$ rotate functions, as shown in Fig.2. IVSPImage provides all the tools for calculating distances, angles, and intersections between $3 \mathrm{D}$ points, lines, and surfaces. These 3D measurement tools can be used to measure the skull model so that accurate asymmetric features of the skull can be obtained.
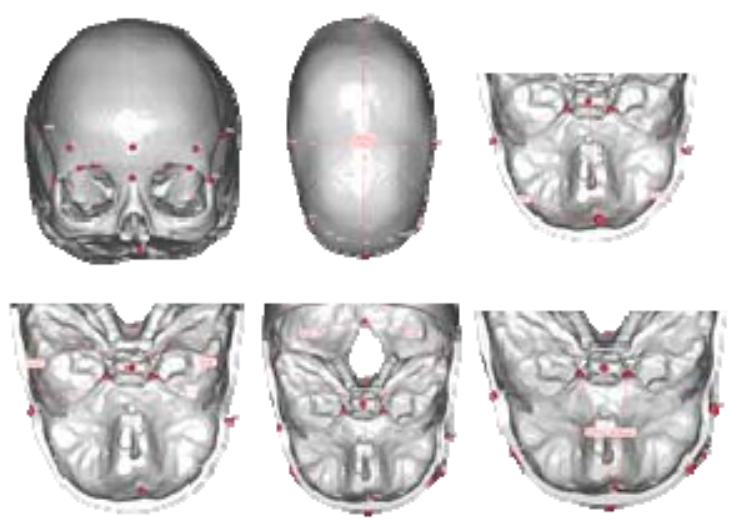

Figure 2 Skull model measurement using 3D software

\section{Step 3. Surgical planning}

Surgical planning is mainly achieved by intersection computing of computer 3D data. The difficulty is that the skull data is derived from true CT data and belongs to reverse engineering data. Compared with the traditional positive data formed by parameter equation, it has the characteristics of big data volume and abundant features. Direct calculations take hours. Therefore, pre-processing of the data and more rapid and stable algorithm selection become the key. In the IVSP Image 3D software, the 3D data is stored via the half-edge data structure to quickly access adjacent point surfaces. Through a series of algorithms such as triangulation, normalization, unified normals, and non-flow pattern detection, general 3D model is transformed into flow pattern data, and intersection operation is greatly accelerated through $3 \mathrm{D}$ BSP tree algorithm. This allows the doctor to simulate the actual osteotomy operation in the software, thus completing the cutting simulation. Fig. 3 


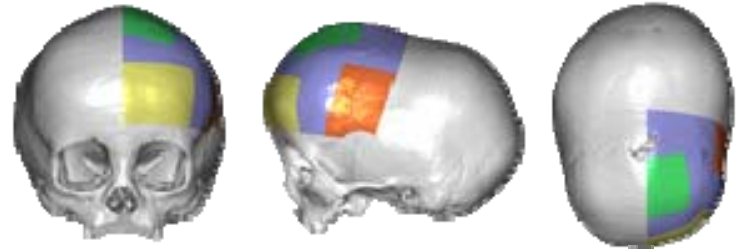

Figure 3 shows osteotomy in practice

After bone block cutting, the bone block needs to be moved to the desired position. Fig 4 . In the software, a series of convenient tools are provided for rotation and movement of bone blocks. At the same time, bone block projections are displayed in real time in different scenes such as 3D scenes and multi-plane reconstruction scenes, so that $3 \mathrm{D}$ difference between bone block position and the expected position can be observed in real time, thus enabling each bone block to reach the ideal 3D position.
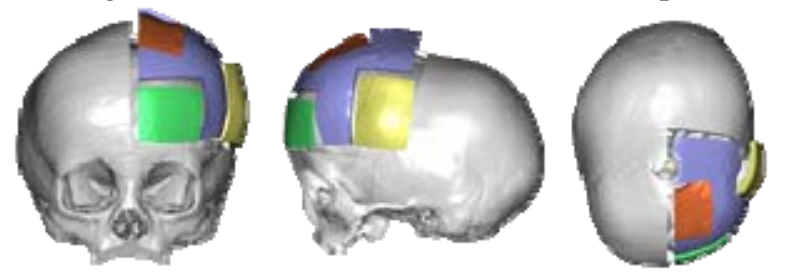

The figure 4 shows the effect of rearranged small bone blocks

\section{Step 4. Comparison before and after operation}

After the surgical planning is completed, by loading the surgical planning model and the preoperative model at the same time, and performing multi-angle and multisection display in the software, one can clearly understand the 3D offset information of the preoperative and planned bone blocks, find scope of bone blocks demanding removal and polishing, as well as important blood vessel and nerve locations, thus reducing the surgical implementation difficulty and avoiding surgical risks, as is shown in Fig. 5, Fig 6.
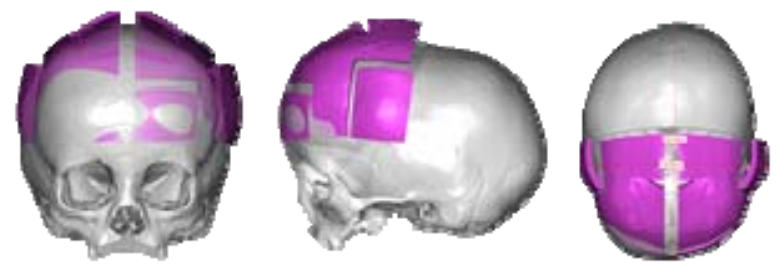

Figure 5 demonstrated multi-angle and multi-section display in the software

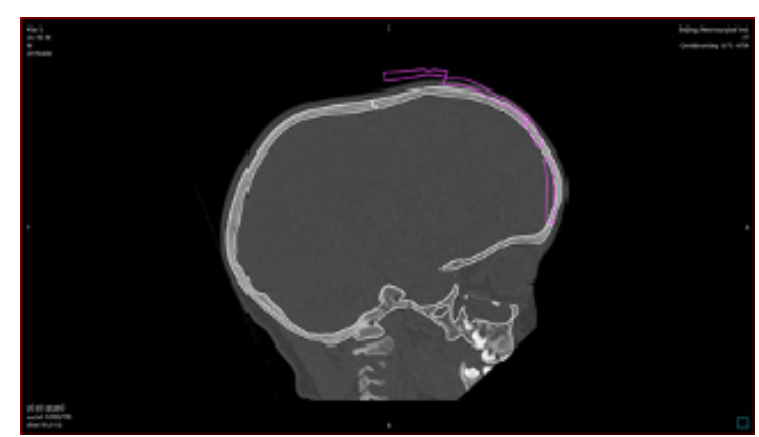

Figure 6 demonstrated the different between preoperative and simulated postoperative skull model

\section{Conclusion}

The use of computer 3D digital technology for preoperative design planning and simulation can reduce surgical difficulty to a certain extent, improve surgical efficiency, significantly increase intraoperative accuracy, and also reduce the risk of intraoperative bleeding and postoperative complications. Different bone block cutting and combining methods can be repeatedly simulated before surgery, so it is easier to acquire the optimal effect in postoperative cranial cavity enlargement and more aesthetic skull shape. In addition, the use of $3 \mathrm{D}$ digital technology also provides a more quantitative objective standard for the diagnosis and evaluation of craniosynostosis, so that more accurate evaluation in postoperative follow-up is possible.

\section{Acknowledgment}

This study is supported by Key Project of International Cooperation on Science and Technology Innovation Among Governments of National Key Research and Development Plan (2017YFE0121200)

Li Xiang and Lu Zheng contributed equally as first authors

\section{References}

1. J. Greenwood, P. Flodman, K. Osann et al . Genet Med. 16 (4) :302-310 (2014)

2. S.A. Rasmussen, M.M. Yazdy, S.L. Carmichael, et al . ObstetGynecol. 110(2Pt 1): 369 - 377 (2007)

3. M. Czerwinski, R.A. Hopper, J.A. Fearon, et al . Plast Reconstr Surg. 126(1): 181-186 (2010)

4. J. Weinzweig, S.B. Baker, L.A. Whitaker, et al. Plast Reconstr Surg. 110(2): 397-408 (2002)

5. D. Renier, E. Lajeunie, E. Arnaud, et al . Childs Nerv Syst. 16(10/11):645 -658 (2000)

6. R.C. Di, G. Paternoster, M. Caldarelli, et al . Childs Nerv Syst. 28(9): 1413-1422 (2012)

7. Shen Weimin, Wang Gang, Cui Jie, et al. Chinese Journal of Plastic Surgery, 23 (4): 284-287 (2007)

8. Xie Xupeng, Shi Yawei, Wu Jiang, et al. Chinese Journal of Clinical Neurosurgery. 13 (1): 50-51 (2008)

9. Zhang Disheng. Craniofacial surgery [M]. Shanghai: Shanghai Science and Technology Press. 2228(1997)

10. Mark. Handbook of Neurosurgery [M]. Greenberg: sixth edition. New York: Thieme. 99-101 (2006)

11. S. Girod, M. Teschner, U. Schrell, et al. Journal of Cranio-Maxillofacial Surgery. 29(3): 156-158 (2001)

12. M. J. Durst. In: Computer Graphics 22(2): $72-$ 73(1988)

13. W. E. Lorensen and H. E. Cline. Computer Graphics 21(3): 163-169 (July 1987) 
14. W.C. Thibault, B.F. Naylor. SIGGRAPH Comput.

Graph. 21(4): 153-162(1987) 